into eight food groups, based on the DASH diet. The pictures of food items were useful to remind patients of the components of food groups. As a result, FFQ was quickly applied, in approximately 20 and 16 min for FFQ at 30 and for 7 days, respectively.

Conclusion This first step allowed detecting that the FFO for food groups was feasible at seven and 30 days, and no major issues were detected during the testing.

\section{P1-506 MULTILEVEL MODELLING OF SURVEY DATA}

doi:10.1136/jech.2011.142976g.94

$1,2 \mathrm{~S}$ Rozi, ${ }^{1} \mathrm{~S}$ Mahmud, ${ }^{2} \mathrm{G}$ Lancaster. ${ }^{1}$ Aga Khan University, Karachi, Pakistan; ${ }^{2}$ Lancaster University, Lancaster, UK

Introduction Presently, about five million people worldwide die yearly from tobacco related diseases. The WHO currently estimates that there are 1.3 billion people who are regular smokers.

Objectives of data analysis To develop two level random effects logistic regression model for the analysis of clustered binary responses to identify factors associated with smoking among school going male adolescents. To assess if the variability between schools is different for the public and private schools using a random coefficient model. Two random effects will account for the variability between public and private schools respectively. To fit a contrasting Generalising Estimating Equation model to deal with two level clustered data for binary outcome. To compare and evaluate the results from the above mentioned models with a conventional logistic regression model as used in the original study.

Methods A two-stage cluster sampling with stratification based on school type was employed for the selection of schools and students. We interviewed 772 male secondary school students. The outcome variable is smoking status of the students. We have two level data with a single level of clustering.

Results Final multilevel random effect model showed that between cluster variance is significantly different from zero ( $\mathrm{p}$ value of likelihood ratio test $=0.01$ ), which indicates that there is variability between schools and we need to take between cluster variation into account by using multilevel modelling. The Intra-class Correlation quantifies consistencies among observations within each cluster and it is also greater than zero (ICC $=0.15$ ).

\section{P1-507 POLYMORPHISMS IN GENES RELATED TO SEX STEROID TRANSPORT AND SIGNALLING MODULATE MENOPAUSAL HORMONE THERAPY EFFECT ON RISK OF COLORECTAL CANCER}

doi:10.1136/jech.2011.142976g.95

${ }^{1} A$ Rudolph, ${ }^{*}{ }^{2} J$ Sainz, ${ }^{1} \mathrm{R}$ Hein, ${ }^{3} \mathrm{M}$ Hoffmeister, ${ }^{3} \mathrm{~B}$ Frank, ${ }^{2} \mathrm{~A}$ Försti, ${ }^{2} \mathrm{~K}$ Hemminki, ${ }^{3} \mathrm{H}$ Brenner, ${ }^{1} \mathrm{~J}$ Chang-Claude. ${ }^{1}$ Division of Cancer Epidemiology, German Cancer Research Center, Heidelberg, Germany; ${ }^{2}$ Division of Molecular Genetic Epidemiology, German Cancer Research Center, Heidelberg, Germany; ${ }^{3}$ Division of Clinical Epidemiology and Ageing Research, German Cancer Research Center, Heidelberg, Germany

Introduction Menopausal hormone therapy (MHT) has been associated with reduced colorectal cancer (CRC) risk. Since the underlying biological mechanisms of MHT effects on CRC are unknown, we investigated whether single nucleotide polymorphisms (SNPs) in genes related to sex steroid metabolism, transport and signalling modify MHT-associated CRC risk.

Methods 47 SNPs in 16 candidate genes related to sex steroid transport (ABCB1), metabolism (COMT, CYP1A1, CYP1A2, CYP1B1, CYP2C9, CYP2C19, CYP3A4, CYP17A1, GSTP, HSD17B1) and signalling (ESR1, ESR2, SHBG, PGR, NR1I2) were genotyped using genomic DNA samples from 685 female postmenopausal CRC patients and 684 controls without CRC of a German populationbased case-control study (DACHS). Unconditional multivariate logistic regression was performed and effect modification was assessed using a multiplicative interaction term.

Results CRC risk associated with ever MHT use as well as with duration was significantly modified by rs1202168 in ABCB1 ( $p$ interaction $=0.04$ ). The MHT-associated risk reduction was no longer significant in homozygous non-carriers, while homozygous carriers of the minor $\mathrm{T}$ allele had a $57 \%$ lower risk with ever use of MHT and a $22 \%$ lower risk per 5 years of MHT use. Significant effect modification was also observed for rs910416 in ESR1, whereby the decreased CRC risk was attenuated in carriers of the minor $\mathrm{C}$ allele ( $p$ interaction $=0.03$ for ever use and 0.07 for duration of use).

Conclusion Our results provide the first evidence that polymorphisms in sex steroid-related genes may modify CRC risk associated with MHT. Our findings warrant replication in independent study populations.

\section{P1-508 OESTROGEN RECEPTOR $\beta$ EXPRESSION IN COLORECTAL CANCER}

doi:10.1136/jech.2011.142976g.96

${ }^{1} \mathrm{~A}$ Rudolph, ${ }^{* 2,3} \mathrm{C}$ Toth, ${ }^{1} \mathrm{R}$ Hein, ${ }^{4} \mathrm{M}$ Hoffmeister, ${ }^{2,3} \mathrm{E}$ Herpel, ${ }^{2} \mathrm{H}$ Bläker, ${ }^{4} \mathrm{H}$ Brenner, ${ }^{1} \mathrm{~J}$ Chang-Claude. ${ }^{1}$ Division of Cancer Epidemiology, German Cancer Research Center, Heidelberg, Germany; ${ }^{2}$ Department of General Pathology, Institute of Pathology, Heidelberg, Germany; ${ }^{3}$ NCT Tissue Bank, National Center for Tumor Diseases (NCT), Heidelberg, Germany; ${ }^{4}$ Division of Clinical Epidemiology and Ageing Research, German Cancer Research Center, Heidelberg, Germany

Introduction The oestrogen receptor $\beta(\operatorname{ER} \beta)$ is the primarily expressed ER in the large intestine and has been found to have inhibitory effects on colon cancer cells. We therefore investigated, whether the ER $\beta$ expression in tumour tissue from colorectal cancer (CRC) patients is associated with tumour/clinical characteristics.

Methods We used tissue micro arrays consisting of paired normal and tumorous tissue samples from 1262 CRC cases of a German population-based case-control study (DACHS). ER $\beta$ expression was measured via immunohistochemistry. Multinomial logistic regression was used to assess the association of ER $\beta$ expression with tumour/clinical characteristics.

Results Measurement of ER $\beta$ expression was successful in 1101 patients. ERß was strongly expressed ( $>50 \%$ positive cells) in normal intestinal tissue, whereas high ER $\beta$ expression was found in $16 \%$, moderate expression in $29 \%$ and no expression in $42 \%$ of tumour samples. Strong vs no expression of ER $\beta$ was inversely associated with higher UICC stages (stage II OR $=0.42,95 \% \mathrm{CI}(0.26$ to 0.66$)$; stage III $\mathrm{OR}=0.40,95 \% \mathrm{CI}(0.25$ to 0.65$)$; stage IV $\mathrm{OR}=0.52,95 \% \mathrm{CI}$ (0.29 to 0.92$) ; \mathrm{p}$ trend $=0.01)$. Furthermore, cases with strong ER $\beta$ expression had a significantly smaller tumour extent (T2 OR $=0.51,95 \% \mathrm{CI}(0.24$ to 1.10$)$; T3 OR=0.24, $95 \% \mathrm{CI}$ (0.11 to 0.49$)$; $\mathrm{T} 4 \mathrm{OR}=0.24,95 \%$ CI $(0.10$ to 0.60$)$; $\mathrm{p}$ trend $<0.001)$ than cases with no expression. No significant associations were seen with histopathological grading, nodal status, distant metastasis, tumour localisation, neoadjuvant treatment, age or sex.

Conclusion We observed an association between high ER $\beta$ expression and smaller tumour extent and confirmed previous reports of an association between high ER $\beta$ expression and less advanced tumour stages.

\section{P1-509 STUDY AETIOLOGY OF LACTATION FAILURE IN NEONATAL AFFECTED BY HYPERNATREMIC DEHYDRATION: A CASE CONTROL STUDY}

doi:10.1136/jech.2011.142976g.97

${ }^{1}$ S Salarilak, ${ }^{2}$ Z Fakour, ${ }^{1} \mathrm{H}$ Zanjani. ${ }^{1}$ Islamic Azad University, Tabriz Branch, Tabriz, East Azerbijan, Iran; ${ }^{2}$ Urmia Medical University, Urmia, West Azerbijan, Iran

Background Inadequate milk supply in exclusively breastfed neonates can lead to neonatal hypernatremic dehydration in the first 